\title{
Higiene das mãos em tempos de pandemia
}

\author{
Hand hygiene in times of pandemic \\ Higiene de las manos en tiempos de pandemia
}

Rozemy Magda Vieira Gonçalves ${ }^{1 *}$, Terezinha de Fátima Gorreis ${ }^{1}$, Rosane Maria Sordi ${ }^{1}$, Elisangela Souza ${ }^{1}$, Nicole Hertzog Rodrigues ${ }^{2}$.

\section{RESUMO}

Objetivo: Analisar através de uma revisão narrativa sobre a prática de higienizar as mãos no tempo de pandemia. Revisão bibliográfica: A higiene de mãos é uma prática de extrema importância para os indivíduos, pois sua ausência ou realização de maneira inadequada pode trazer sérias consequências aos pacientes e a sociedade em geral. Em relação à higiene de mãos associada ao contexto da pandemia de COVID-19, é notório que as práticas tenham novamente recebido um incremento, visto a magnitude e periculosidade da doença. Tornar a lavagem das mãos parte dos programas de desenvolvimento é uma forma econômica de reduzir a transmissão de doenças, prevenir pandemias e ajudar a alcançar os muitos objetivos de desenvolvimento sustentável. Considerações finais: Além das medidas emergenciais, devem ser adotadas providências que mantenham uma prática sustentada de higiene de mãos como uma das medidas de prevenção de infecção por Coronavírus ou por outras infecções relacionadas à saúde. As intervenções devem ser projetadas com a finalidade de serem específicas ao contexto vivenciado, inclusivas e atraentes, motivando as pessoas e mudando seu ambiente físico para incentivar e reforçar comportamentos.

Palavras-chave: Higiene das mãos, Pandemia, COVID-19.

\begin{abstract}
Objective: Analyze through a narrative review on the practice of hand hygiene during the time of a pandemic. Bibliographic review: Hand hygiene is an extremely important practice for individuals, as its absence or improper performance can bring serious consequences to patients and society in general. Regarding hand hygiene associated with the context of the COVID-19 pandemic, it is notable that practices have again received an increase, given the magnitude and dangerousness of the disease. Making hand washing a part of development programs is an economical way to reduce disease transmission, prevent pandemics and help achieve the many sustainable development goals. Final considerations: In addition to emergency measures, measures must be taken to maintain a sustained hand hygiene practice as one of the measures to prevent infection by Coronavirus or other health-related infections. Interventions should be designed to be specific to the context experienced, inclusive and attractive, motivating people and changing their physical environment to encourage and reinforce behaviors.
\end{abstract}

Key words: Hand hygiene, Pandemic, COVID-19.

\section{RESUMEN}

Objetivo: Analizar a través de una revisión narrativa sobre la práctica de la higiene de las manos durante la época de una pandemia. Revisión bibliográfica: La higiene de manos es una práctica de extrema importancia para los individuos, ya que su ausencia o mal desempeño puede traer graves consecuencias para los pacientes y la sociedad en general. En cuanto a la higiene de manos asociada al contexto de la pandemia

${ }_{1}^{1}$ Hospital de Clínicas de Porto Alegre (HCPA), Porto Alegre - RS. *E-mail: rozygong@gmail.com

2 Universidade Federal de Ciências da Saúde de Porto Alegre (UFCSPA), Porto Alegre - RS.

SUBMETIDO EM: 6/2021

ACEITO EM: 6/2021

PUBLICADO EM: 6/2021 
de COVID-19, se destaca que las prácticas han vuelto a recibir un incremento, dada la magnitud y peligrosidad de la enfermedad. Hacer que el lavado de manos forme parte de los programas de desarrollo es una forma rentable de reducir la transmisión de enfermedades, prevenir pandemias y ayudar a lograr los numerosos objetivos de desarrollo sostenible. Consideraciones finales: Además de las medidas de emergencia, se deben tomar medidas para mantener una práctica sostenida de higiene de manos como una de las medidas para prevenir la infección por Coronavirus u otras infecciones relacionadas con la salud. Las intervenciones deben estar diseñadas para ser específicas al contexto experimentado, inclusivas y atractivas, motivando a las personas y cambiando su entorno físico para alentar y reforzar comportamientos.

Palabras clave: Higiene de las manos, Pandemia, COVID-19.

\section{INTRODUÇÃO}

Profissionais de um hospital de grande porte, vem vivenciado, dia a dia, mudanças nas práticas relacionadas à lavagem de mãos, envolvendo a equipe de saúde e a população circulante dentro do hospital. Para promover uma mudança social relativa a uma melhor Higiene de Mãos (HM), acredita-se ser necessário que uma boa parcela de um grupo adote melhores práticas relacionadas a HM, levando aos demais indivíduos à aplicação sustentada deste hábito. As mãos são consideradas ferramentas essenciais de trabalho para os profissionais de saúde e as principais vias de disseminação de infecções (GAUTAM OP, 2020; GONÇALVES RMV, 2013).

A partir do surto da Gripe A em 2009, ocorreu uma severa mudança em relação às práticas de higienização de mãos, não somente nos serviços de saúde, mas também em estabelecimentos de grande circulação pública que incluía a obrigatoriedade de disponibilização de álcool $70 \%$ em gel para a antissepsia das mãos (GONÇALVES RMV, 2013). Já em relação a HM associada ao contexto da COVID-19, é notório que as práticas de HM tenham novamente recebido um incremento, visto a magnitude e periculosidade da doença (AZEVEDO AP, et al., 2021).

De acordo com Gautam OP (2020), para a contenção de futuras pandemias, a HM deve ser colocada em foco através de programas que incentivem e provoquem a adesão a esse hábito, a longo prazo. Assim, outras doenças transmissíveis, surtos e novas pandemias serão prevenidas. Entidades como governos, setores privados e públicos, assim como universidades e sociedade civil devem atuar em colaboração em busca dessa mudança de comportamento favorecendo às práticas de higiene, incluindo a HM. Também, medidas de vigilância e controle devem ser implementadas para avaliar, monitorar e prever melhorias por meio de programas de intervenções (CHEN X e YU B, 2020).

Frequentemente, existe a crença de que as vacinas são a única opção para tratamento de doenças contagiosas, e servirão como uma solução simples e com grande eficiência frente a um problema complexo. O clamor pela vacina contra a COVID-19 vem contextualizado pelo imediatismo de sentimentos de medos e incertezas, mas provavelmente não cessará o fenômeno da hesitação vacinal de forma mais ampla (HARRISON EA e WU JW, 2020). Vivenciamos na pandemia global da COVID-19 que a liberdade individual não é absoluta quando contraposta às necessidades essenciais das coletividades (GOSTIN LO e WILEY LF, 2020).

Sabe-se que nem todas as vacinas são 100\% eficazes, portanto, a higiene das mãos prevalecerá. Mantendo a higienização, principalmente a HM, evita-se que grande maioria dos vírus (incluindo bactérias e fungos), adentrem ao corpo humano. Portanto, a prática de higiene das mãos associada às vacinas, deve obter incremento financeiro, principalmente, frente a uma pandemia. Ainda, a HM é uma prática valiosa independente se se terem ou não, vacinas que previnam a COVID-19, pois a longo prazo, às vacinas associadas a HM terão efeito somatório frente ao controle da propagação de doenças e de novas pandemias (GAUTAM OP, 2020).

Dessa forma, o objetivo do presente estudo foi analisar através de revisão narrativa a HM em tempos de pandemia. 


\section{REVISÃO BIBLIOGRÁFICA}

\section{Higiene das mãos em tempos de pandemia}

No passado, quando havia grandes ameaças nacionais à saúde, como o alastramento de doenças infecciosas, o governo federal destinava fundos de doações e programas que seriam usados em intervenções na saúde da população. Esses recursos destinavam-se a fornecer uma força de trabalho humana treinada e capacitada para conter disseminações. Neste contexto de qualificação, sabe-se que os sistemas de monitoramento eletrônico têm o potencial de melhorar a conformidade com a HM para prevenir infecções associadas à saúde, porém, os custos são elevados (SRIGLEY JA, et al., 2013; SRIGLEY JA, et al., 2015).

Para tal, se houver financiamento disponível as instalações de saúde, com maiores subsídios financeiros ou programas governamentais, seria ideal utilizar-se alguns desses recursos em métodos de vigilância, como o uso da tecnologia de monitoramento eletrônico que melhoraria a conformidade com a $\mathrm{HM}$, através de intervenções imediatas (CHEN X e YU B, 2020).

Esses sistemas ajudam a remover o efeito Hawthorne (observações diretas in loco), que promove a mudança de comportamento quando os indivíduos estão sendo observados diretamente na aplicabilidade de alguma técnica ou função, principalmente quando essa função é avaliativa, e o viés de observação associado aos programas de cliente secreto. Mas, em última análise, a tecnologia não pode resolver todas as barreiras para a conformidade com a HM e para realmente termos uma mudança sustentada, uma mudança cultural deve ocorrer dentro da instalação de saúde (SOUZA LM, et al., 2015).

Paula DG, et al. (2017) citam que, modelagem de funções, o estado da arte (ações conjuntas à aplicação de lembretes), atividades educativas, feedback oportuno, produtos e instalações adequadas, monitoramento consistente e relatórios de dados ajudam a apoiar a cultura de mãos limpas, se fazem necessários nas instalações de saúde.

Conforme Brasil (2015) e Gonçalves RMV (2013), mencionaram que durante o evento da Gripe A a prática de HM foi amplamente disseminada nos espaços escolares além de outros lugares públicos e privados, sugerindo que a HM se tornou naquele momento uma prática de constante vigilância onde, às pessoas passaram a carregar consigo, o seu próprio frasco com álcool gel $70 \%$, o que vem se repetindo de certa forma na atual pandemia. Mas, assim como essa prática, após o controle do H1N1 caiu no esquecimento para o público em geral, pode também ser negligenciada, meses após o controle da pandemia da COVID-19.

Antes que os hábitos voltem aos modos pré-COVID-19, as medidas educativas têm a oportunidade de enaltecer e renovar a HM na área da saúde a fim de estimular e orientar o autocuidado e, dessa forma, melhorar a adesão a HM, além de consolidar o pensamento crítico sobre o risco biológico (PAULA DG, et al., 2017).

A mudança de comportamento durante uma pandemia está relacionada a vários fatores: avaliação de risco, medos pessoais, determinantes sociais, campanhas informativas e expectativas culturais. Os determinantes sociais da saúde estão relacionados às condições em que uma pessoa vive e trabalha. Sendo assim, podem interferir diretamente nos reflexos da pandemia, tanto positivamente quanto negativamente (JACQUELINE B e HAENDEL G, 2020).

Para impedir a propagação da COVID-19, juntamente com outros comportamentos apropriados, a prática de HM em intervalos regulares é imprescindível após tossir ou espirrar, ao cuidar de doentes, após usar o banheiro, antes de comer, durante o preparo de alimentos após manusear animais ou resíduos. Lavar as mãos depois de tocar em superfícies comuns, ou depois de voltar para casa de uma visita a um local público, manterá a segurança de nós e de outras pessoas ao nosso redor (OPAS, 2020).

Deve-se desenvolver medidas visando à maior utilização dessa rotina básica de prevenção de infecções hospitalares pela equipe multiprofissional em saúde. $O$ ato de higienizar as mãos, conforme preconizado, consiste no primeiro passo para a busca da segurança e da excelência na qualidade da assistência ao paciente (AZEVEDO AP, et al., 2021). 
A não realização dessa prática compromete o profissional, o paciente e outros que venham a ter contato com eles, e também os objetos os quais foram contaminados. A prática sustentada de HM pelos profissionais de saúde no momento certo e de maneira correta, propicia na minimização da disseminação de infecções no ambiente hospitalar e suas repercussões (SOUZA LM, et al., 2015).

Um desafio para compreender como os hábitos de HM mudaram na área de saúde durante a COVID-19, é a capacidade de medir e relatar dados de conformidade com precisão. A HM é normalmente monitorada por meio de observações secretas de clientes que nem sempre são confiáveis, podendo causar uma lacuna no conhecimento para entender se a conformidade aumentou ou se isso é uma percepção ou suposição (SRIGLEY JA, et al., 2013).

Durante a pandemia, a equipe que normalmente realiza essas observações, podem estar redirecionadas para outras funções na assistência aos doentes, ou não estarem disponíveis por problemas de saúde ou de ordem pessoal. Monitorar e observar a HM pode não ser uma prioridade quando outras preocupações com o atendimento ao paciente são primordiais (BRASIL, 2020).

As instituições de saúde têm lutado para alcançar e manter altas taxas de conformidade com a HM, apesar da consciência e do conhecimento de sua importância por séculos (JACQUELINE B e HAENDEL G, 2020). Se tem conhecimento que o álcool em gel a $70 \%$ é tido na literatura como um meio de se ampliar a adesão dos profissionais de saúde à HM e diminui a taxa de Infecções Relacionadas à Assistência à Saúde (IRAS), visto que se gasta menos tempo para a realização dessa prática, já que o produto age de forma mais rápida e é eficaz na redução da carga microbiana (MEDEIROS KC, et al., 2017; KOTAY SM, et al., 2019).

Dadas tantas razões pelas quais a HM não é realizada, como um preventivo de infecção, é importante reconhecer essas barreiras e tentar abordá-las dentro da organização para garantir que os profissionais de saúde possam se sentir apoiados para fazer o que é certo e ter um ambiente que ampara a HM. Pois os profissionais de saúde avaliam a adesão de HM da categoria profissional a que pertencem como superior a outras, isso pode estar relacionado ao corporativismo, implicando um acordo tácito (PAULA AO e OLIVEIRA AC, 2017).

Já Souza LM, et al. (2015) mencionam que a crença pessoal e os hábitos adquiridos durante a vida podem desempenhar maior influência na adesão da HM do que o conhecimento científico construído profissionalmente. Parte disso inclui o desenvolvimento de uma cultura de segurança dentro da organização que encoraja a conformidade com todos os protocolos e iniciativas de prevenção de infecções.

\section{Higiene das mãos e o COVID-19}

A epidemia causada pelo novo Coronavírus (SARS-CoV-2) tornou-se um problema de saúde pública mundial, propagando-se de forma rápida e tornando-se uma pandemia. Medidas que visem à contenção do vírus são analisadas em todos os países afetados pela infecção, com o intuito de assegurar barreiras que impeçam o aumento do quantitativo de casos de infecção (NASCIMENTO CS, et al., 2020). O SARS-CoV-2 pode ser transmitido de pessoa a pessoa por meio de gotículas respiratórias e contato próximo. Em ambientes de saúde, isso destaca a necessidade de praticar a higiene respiratória e a $\mathrm{HM}$ e usar equipamento de proteção individual adequado (LAI THT, et al., 2020).

A HM com gel à base de álcool é utilizada mundialmente como um dos procedimentos mais eficazes, simples e de baixo custo contra a transmissão cruzada da COVID-19. O álcool inativa o vírus, pois ao acionar a bicamada lipídica que o compõe geneticamente o etanol o dissolve, e as formulações de ABHR com pelo menos $60 \%$ de etanol têm se mostrado eficazes para a HM (LAl THT, et al., 2020). No Brasil, outras formulações foram aprovadas como higienizadores, pela Agência Nacional de Vigilância Sanitária (Anvisa) como: álcool etílico $70 \%$ (p/p, equivalente à relação ponderal em massa) e $77 \%$ (v/v), álcool etílico glicerinado $80 \%$ (v/v), álcool isopropílico glicerinado 75\% (v/v) e álcool etílico em gel (SEQUINEL $R$, et al., 2020).

Promover sempre a lavagem das mãos com água e sabão é uma das intervenções de saúde pública mais econômicas na redução da sobrecarga global das doenças infecciosas. Agora a lavagem das mãos está sendo posta juntamente com outros comportamentos-chave, como a primeira linha de defesa para restringir 
a pandemia da COVID-19 (GAUTAM OP, 2020). Essa doença é disseminada através de gotículas de uma pessoa que tosse ou espirra e através de superfícies contaminadas (GAUTAM OP, 2020; NASCIMENTO CS, et al., 2020).

Os profissionais de saúde devem aderir aos "Meus cinco momentos para HM", conforme a OMS: antes de tocar em um paciente, antes de procedimentos limpos ou assépticos, após exposição a fluidos corporais ou risco, depois de tocar em um paciente e após tocar os arredores do paciente (SOUZA LM, et al., 2015; PAULA DG, et al., 2017). No entanto, os baixos níveis de conformidade com a HM ainda são um desafio constante, apesar das inúmeras intervenções e campanhas realizadas (AZEVEDO AP, et al., 2021; SOUZA LM, et al., 2015).

De acordo com relatórios existentes, a HM é frequentemente negligenciada pelos profissionais de saúde tanto em países desenvolvidos quanto em desenvolvimento, com taxas de conformidade às vezes caindo abaixo de $20 \%$. A baixa adesão a HM não tem ocorrido por falta de conhecimento, mas sim por comportamentos de risco, principalmente em meio a uma pandemia em que a todo momento informações de prevenção estão audíveis e visíveis mundialmente (PAULA AO e OLIVEIRA AC, 2017).

Como a OMS relatou recentemente, mais nove milhões de enfermeiras e parteiras são necessárias em todo o mundo para alcançar a cobertura universal de saúde até 2030 (OPAS, 2020). A enfermagem, pelas próprias características inerentes da profissão, está em contato mais frequente com os pacientes e os tocam seguidamente para a realização dos cuidados básicos de vida, predispondo-os adquirir ou transmitir infecções como a COVID-19 (PAULA AO e OLIVEIRA AC, 2017). A maioria das infecções adquiridas na área de saúde poderiam ser evitadas por enfermeiras bem treinadas com conformidade adequada de $\mathrm{HM}$ e uso de equipamento de proteção (PAULA DG, et al., 2017).

Para garantir a segurança e a qualidade assistencial, é fundamental dispor de pessoal de enfermagem em número adequado e acesso aos equipamentos necessários. Atuações que visam redução de infecções nos ambientes de saúde e a proteção do quadro funcional, por meio de medidas simples e gastos rasos, devem ser analisadas (PAULA DG, et al., 2017). A alta transmissibilidade da COVID-19 põe em alerta a demanda mundial para o implemento de insumos para a prevenção e controle de infecção nos ambientes de saúde (BRASIL, 2020).

A falta de boas práticas de HM permite a transmissão de doenças e surtos, mantendo crianças fora da escola, impedindo os adultos de trabalhar, colocando pessoas mais vulneráveis no hospital e instituições de agrupamentos em risco de serem centros de infecção (GAUTAM OP, 2020).

A Organização Pan-Americana de Saúde (OPAS) e a OMS apoiam também campanhas de interesse aos profissionais de saúde dentro do Primeiro Desafio Global para a Segurança do Paciente, que está focado na prevenção das Infecção Relacionada à Assistência à Saúde (IRAS), sob o lema "Uma Assistência Limpa é Uma Assistência Mais Segura" (SOUZA LM, et al., 2015).

Neste contexto, destaca-se que a lavagem simples das mãos com água e sabão é capaz de reduzir inúmeras infecções como em até $48 \%$ o risco de diarreia endêmica, em até $23 \%$ às infecções respiratórias agudas, em até $50 \%$ os casos de pneumonia, em até $27 \%$ das mortes de crianças e reduziu em até $36 \%$ o risco de contágio por coronavírus sazonal (GAUTAM OP, 2020).

Tornar a lavagem das mãos parte dos programas de desenvolvimento é, portanto, uma forma econômica de reduzir a carga de doenças, prevenir pandemias tanto atuais, quanto futuras e ajudar a alcançar os muitos objetivos de desenvolvimento sustentável para os quais é crucial (GAUTAM OP, 2020).

\section{CONSIDERAÇÕES FINAIS}

A higienização das mãos sempre foi a maneira mais comum de prevenir a disseminação de infecções, a exemplo da COVID-19. Embora pareça uma medida simples e de fácil realização, essa prática tem voltado ao foco durante a pandemia do novo coronavírus, sendo uma prática valiosa por ser econômica e muito eficaz na redução de inúmeras doenças infecto contagiosas. Porém, além das medidas emergenciais, devem ser 
adotadas providências que sustentem o hábito da $\mathrm{HM}$ a longo prazo. A negligência dessa prática por profissionais de saúde configura-se como comportamento de risco e permite a propagação de doenças e surtos sendo um desafio, a aplicação de medidas que mantenham a aderência dos profissionais e da sociedade a este hábito.

\section{REFERÊNCIAS}

1. AZEVEDO AP, et al. Adesão da higienização das mãos entre equipes multidisciplinar em unidades de terapia intensiva de um hospital referência em infectologia. Revista Eletrônica Acervo Enfermagem, 2021; 9: 1-8.

2. BRASIL. Protocolo de Tratamento de Influenza $2015 . \quad$ Disponível em: https://bvsms.saude.gov.br/bvs/publicacoes/protocolo_tratamento_influenza_2015.pdf. Acesso em: 24 de junho de 2021.

3. BRASIL. A Gestão de Risco e Governança na Pandemia por COVID-19 no Brasil 2020. Disponível em: https://portal.fiocruz.br/sites/portal.fiocruz.br/files/documentos/relatoriocepedes-isolamento-social-outrasmedidas.pdf. Acesso em: 29 jun. 2021.

4. CHEN X, YU B. First two months of the 2019 Coronavirus Disease (COVID-19) epidemic in China: real-time surveillance and evaluation with a second derivative model. Global Health Research and Policy, 2020; 5(7): 2-9.

5. GAUTAM OP. Higiene das mãos: crucial para controlar a COVID-19 e prevenir futuras pandemias. 2020. In: Wateraid. Disponível em: https://washmatters.wateraid.org/pt-pt/blog/higiene-das-maos-crucial-para-controlar-a-covid-19-eprevenir-futuras-pandemias. Acesso em: 01 jun. 2021.

6. GONÇALVES RMV. Educação, mídia e saúde: uma análise da construção do pânico e do risco em Zero Hora. Dissertação (Mestrado em Educação). Universidade Luterana do Brasil, Canoas, 2013; 132p.

7. GOSTIN LO, WILEY LF. Governmental Public Health Powers During the COVID-19 Pandemic: Stay-at-home Orders, Business Closures, and Travel Restrictions. JAMA, 2020; 323(21): 2137-2138.

8. HARRISON EA, WU JW. Vaccine confidence in the time of COVID-19. European Journal of Epidemiology, 2020; 35(4):325-330.

9. JACQUELINE B, HAENDEL G. Ignaz semmelweis: as lições que a história da lavagem das mãos ensina. 2020. In: Fundação Oswaldo Cruz. Disponível em: ttps://agencia.fiocruz.br/ignaz-semmelweis-licoes-que-historia-da-lavagemdas-maos-

ensina\#: :text=Ignaz\%20Semmelweis\%3A\%20as\%20li\%C3\%A7\%C3\%B5es\%20que\%20a\%20hist\%C3\%B3ria\%20 da\%20lavagem $\% 20$ das $\% 20 \mathrm{~m} \% \mathrm{C} 3 \%$ A3os\%20ensina,-

Imprimir\&text=Lavar\%20as\%20m\%C3\%A3os.,Sars\%2DCov\%2D2). Acesso em: 1 jun. 2021.

10. KOTAY SM, et al. Droplet-Rather than aerosol-mediated dispersion is the primary mechanism of bacterial transmission from contaminated hand-washing sink traps. Applied and environmental microbiology, 2019; 85(2): e01997-18.

11. LAI THT, et al. Reply to "Does hand hygiene reduce SARS-CoV-2 transmission?" Graefe's Archive for Clinical and Experimental Ophthalmology, 2020; 258: 1135.

12. MEDEIROS KC, et al. Higienização das mãos entre profissionais de enfermagem circulantes de sala operatória. Revista enfermagem atual in derme, 2017; 81(19): 63-69.

13. NASCIMENTO CS, et al. Medidas de contenção do vírus Sars-CoV-2 em tempos pandêmicos: uma questão de saúde pública. Revista Eletrônica Acervo Enfermagem, 2020; 6(1): 1-8.

14. ORGANIZAÇÃO PAN-AMERICANA DE SAÚDE (OPAS). OMS define 2020 como o internacional dos profissionais e obstetrícia. Brasília: Distrito Federal. 2020. Disponível em: https://www.paho.org/pt/noticias/3-1-2020-oms-define2020-como-ano-internacional-dos-profissionais-enfermagem-e-obstetricia. Acesso em: 01 jun. 2021.

15. PAULA AO, OLIVEIRA AC. A percepção dos profissionais de saúde em relação à higienização das mãos. Revista de Pesquisa Cuidado é Fundamental Online, 2017; 9(2): 321-326.

16. PAULA DG, et al. Estratégias de adesão à higienização das mãos por profissionais de saúde. Revista de Epidemiologia e Controle de Infecção, 2017; 7(2): 113-121.

17. SEQUINEL R, et al. Soluções a base de álcool para higienização das mãos e superfícies na prevenção da COVID19: compêndio informativo sob o ponto de vista da química envolvida. Química Nova, 2020; 43(5):679-684.

18. SOUZA LM, et al. Adherence to the five moments for hand hygiene among intensive care professionals. Revista Gaúcha de Enfermagem, 2015; 36(4): 21-28.

19. SRIGLEY JA, et al. Hand hygiene monitoring technology: protocol for a systematic review. Systematic Reviews, 2013; 2(101): 1-8.

20. SRIGLEY JA, et al. Tecnologia de monitoramento de higiene das mãos: uma revisão sistemática da eficácia. The Journal of hospital infection, 2015; 89 (1): 51-60. 